\title{
The influence of ion implantation by phosphorous on structural changes in porous silicon
}

\author{
Z. Swiatek ${ }^{1}$, I. Lytvynchuk ${ }^{2}$, I. Fodchuk ${ }^{2}$ \\ ${ }^{1}$ Institute of Metallurgy and Materials Science, Krakow, Poland \\ ${ }^{2}$ Chernivtsi National University, Chernivtsi, Ukraine
}

\begin{abstract}
Structural changes in the surface layer of technologically treated silicon by ion implantation, chemical etching, and their combined action have been investigated by the Xray diffractometry methods. The functional and quantitative differences in the thickness dependences of strains, values of maximum strain, level of lattice disturbance and extension of elastic strains nave been revealed after different steps of treatment. The essential modification of photoluminescence spectra was observed in the porous layer after implantation by phosphorus ions in the process of natural aging.
\end{abstract}

Keywords: ion implantation, porous silicon, photoluminescence, X-ray diffraction.

Paper received 13.08.04; accepted for publication 21.10.04.

\section{Introduction}

Porous silicon represents the c-Si single crystal, in which the extensive set of small pores is created by the chemical etching. The density of pores in some samples can be so large, that there is their overlapping, and the unetched parts of silicon acquire the form of the coral-like system of filaments of variable cross-sections [1-6].

In view of the perspective of porous silicon for practical using at the creation of silicon light-emitting devices it is necessary to intensive study of porous silicon properties. This is due to the fact that on the way of realization of similar devices there are serious problems related to instability of structural, optical, luminescent and other characteristics of porous silicon. The one of the supposed solutions of this problem is using the ion implantation and certain modes of heat treatment [7-10]. However, post-implantation defects formed in the result of ion implantation and annealing do not give the possibility to use in full measure the electric and structural proporties of amorphous surface layer of material. The presence of local strains in the transitional gap of $\mathrm{Si}_{a m} / \mathrm{Si}_{c r}$ heteroboundary often results in the displacement of absorption bands [8].

The purpose of this work is to research structural changes in surface layers of the silicon exposed to different types of technological treatments (ion implantation, chemical etching and their combined action) by the methods of X-ray diffractometry and atomic force microscopy.

\section{Object of researches}

To determine the structural changes arising due to different technological treatments of (111) surface, a sample of the high-perfect Czochralsky grown silicon was used. This sample was exposed to the chemical etching and polishing. Thereafter the chemical cleaning of surface was carried out. Four regions were created on the sample surface (corresponding designations are shown in Fig. 1): the first was initial material, second and third were exposed to ion implantation, third and fourth were exposed to the chemical etching in the $\mathrm{HF}, \mathrm{HNO}_{3}$ solution for 40 seconds. Due to chemical etching a porous structure was formed in third and fourth regions. Moreover, the third region was additionally exposed to ion implantation by phosphorus before etching. The energy of implanted ions was $E=180 \mathrm{keV}$, dose was about $Q=8 \cdot 10^{14} \mathrm{ion} / \mathrm{cm}^{2}$. Implantation was carried out in such a way that the ion channeling was impossible. At these doses and energies of implanted ions a surface layer was destroyed with formation of numerous disordered regions with the considerable concentrations of point defects, which caused the changes of material optical properties [8-10].

\section{Experimental part}

The symmetric, asymmetric and skew-asymmetric Bragg geometries of X-ray diffraction with (111), (333), (311) 


\section{Z. Swiatek et al.: The influence of ion implantation by phosphorous on ...}
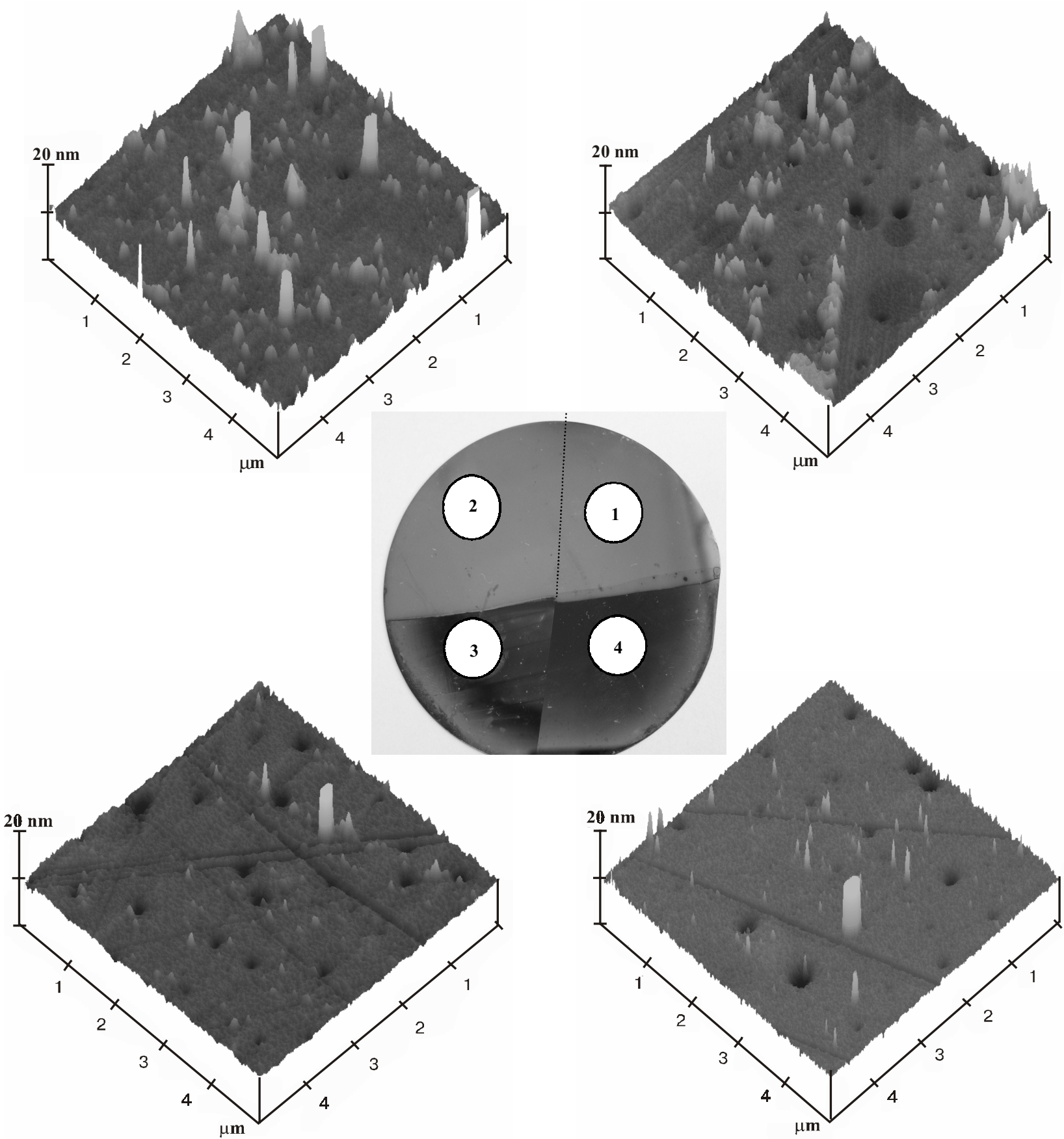

Fig. 1. Atomic-force microscopy (AFM): part №1 is initial material; part №2 implanted by P ions; part №3 implanted by $\mathrm{P}$ ions and chemically etched (porous part); part №4 chemically etched (porous part).

reflections of $\mathrm{CuK}_{\alpha}$ radiation were used for X-ray diffractometry and topography researches.

$\mathrm{X}$-ray rocking curves were measured using a three-axis diffractometer. Two germanium monochromators were placed on the first two axes of diffractometer and the investigated silicon crystal was located on the third axis.
The monochromators in mutually dispersion arrangement with the symmetric (333) reflection practically suppressed $\pi$-polarization of the characteristic $\mathrm{CuK}_{\alpha 1}$ radiation. Highly collimated incident beam had the following spectral and spatial charachteristics: $\frac{\Delta \lambda}{\lambda} \approx 5 \cdot 10^{-7}$ and $\delta \theta=2^{\prime \prime}$. 


\section{Z. Swiatek et al.: The influence of ion implantation by phosphorous on ...}

\section{Theoretical part}

Simulation of X-ray diffraction patterns for crystal with the damaged surface layer is carried out using kinematic and dynamic theories of X-ray scattering. In the dynamic theory contrary to kinematic, the effects of multiple scattering are taken into account, and therefore this theory devoid of limitations on the layer thickness. Thus it is effectively used for the analysis of rocking curves obtained from crystals exposed to different external influences [11, 14-15].

It should be noted that using skew-asymmetric diffraction for certain reflective planes and rotating crystal around the normal to the reflective plane we can gradually decrease extinction depth by more than two orders [16]. Its magnitude can be smaller or comparable with the effective thickness of the damaged surface layer.

The rocking curves were calculated within the framework of the kinematic theory for X-ray reflection. By the functional definition of strain profile $\varepsilon(z)=\Delta d(z) / d$ and distortions of the surface layer $W(z)\left(W=1-e^{L}\right.$, where $L$ is the Debye-Waller factor), it was achieved a satisfactory agreement with the experiment. The functional dependences $\varepsilon(z)$ and $W(z)$ were specified in the form of a set of exponential and sinusoidal maxima with various heights and widths located at the determined depths. At the same time, the ordinate of each point of rocking curves is the function of the whole strain profile $\varepsilon(z)$. A first approximation of this strain profile is determined by a solution of the inverse problem of X-ray diffraction using experimental rocking curves [14, 17].

Within the framework of the kinematic theory the amplitude of X-rays reflected from $N$ layers is the sum of amplitudes reflected from each $j$-th layer and corrected for the phase shift caused by $(\Delta d / d)_{j}$ and $W_{j}$. It is given by [17]:

$$
A_{N}=|q| \sum_{j=1}^{N} e^{-L j} \cdot \exp \left\{i\left[(n-1) \frac{\delta_{j}}{2}+\Phi_{j}\right]\right\} \cdot \frac{\sin \left(\frac{n \delta}{2}\right)}{\sin \left(\frac{\delta}{2}\right)}
$$

where $q=i \frac{d \lambda}{V \sin \theta_{B}} \cdot \frac{e^{2}}{m c^{2}} \cdot\left|F_{h k l}^{o}\right|$ is the amplitude of wave reflected from the single crystallographic plane of perfect crystal in the case when the amplitude of the incident wave is equal to $1 ; V$ is the unit cell volume; $\delta_{j}$ is the phase diference between reflected beams from neighbouring crystallographic planes in the $j$-th layer:

$\delta_{j}=\frac{4 \pi}{\lambda} \cdot d \cos \theta_{B} \cdot \Delta \theta+2 \pi \cdot(\Delta d / d)_{j} ;$

$\Phi_{j}$ is the total phase shift after transmission through the $j-1$ layers:

$$
\Phi_{j}=\sum_{i=1}^{j-1} n_{i} \delta_{j}, \Phi_{1}=0
$$

The Debye-Waller factor is given by the expression:

$L_{j}=\frac{8 \pi^{2}}{\lambda^{2}} \cdot \sin ^{2} \theta_{B} \cdot \bar{U}_{j}^{2}$,

where $\lambda$ is the radiation wavelength, $\bar{U}_{j}^{2}$ is the root-mean square displacement of lattice sites in the $j$-th layer caused by the defects of structure. Distortions $W_{j}=1-e^{-L_{j}}$ are equal to 0 in the $j$-th layer for a perfect crystal and to 1 in the case of the fully disordered lattice.

To obtain a satisfactory agreement between theoretical and experimental rocking curves, the method of minimization of non-negative discrepancy was used in the case of direct problem solution [13]:

$$
\begin{aligned}
& F\left(x_{1}, x_{2}, \ldots, x_{10}, \Delta \theta\right)= \\
& =\sum_{i=1}^{k} \rho_{k}\left[\frac{I_{\exp }\left(\Delta \theta_{k}\right)-I\left(x_{1}, \ldots, x_{10}, \Delta \theta_{k}\right)}{I_{\exp }\left(\Delta \theta_{k}\right)}\right]^{2},
\end{aligned}
$$

where $\rho_{k}\left(\Delta \theta_{k}\right)=1-\left[1+\left(\Delta \theta_{k}-\theta_{0}\right)^{2} W\right]^{-1}$ is the weight function, $\theta_{k}$ is the abscissa of the $k$-th point in the rocking curve, $\theta_{0}$ is the abscissa of the main maximum corresponding to X-ray scattering from the crystal volume, $W$ is reverse to the halfwidth of the rocking curve for an ideal crystal.

The whole profile of strains was divided into thin layers as well as the profile of distortions. In the first stage of optimization, the values of $(\Delta d / d)_{j}$ and $W_{j}$ were determined in each thin layer. Then variation of the obtained values had been carried out so long as the greatest possible agreement $(<3 \%)$ between theoretical and experimental rocking curves was achieved (Figs 2-4). The experimental data processing was accepted as correct for the chosen strain model, if the value of the fitting criterion $F$ was within the range of $\chi^{2}=1 \pm \sqrt{2 / n_{f}}$ (here $n_{f}=n-n_{p}$ indicates a number of freedom degrees at the fitting, $n$ is a number of experimental points, $n_{p}$ is a number of variable parameters) [17].

It should be noted that computer processing these $\mathrm{X}$-ray rocking curves with the fitting procedure and minimization of discrepancy function does not guarantee the uniqueness of obtained results without the detailed analysis of influence degree of different parameters on the form of rocking curves and precision of determination of these parameters.

\section{Results}

\subsection{X-ray researches}

Quantitative estimations of structural changes in the marked parts of the silicon sample were defined from the analysis of rocking curves for (111) and (333) reflections of $\mathrm{CuK}_{\alpha 1}$-radiation (Figs 2-4).

The analysis of diffraction curves of X-ray reflection in Figs 2-4 shows that the external influences (mentioned above) on the surface of the porous Si crystal produce the changes of the rocking curve form especially its "tails" 


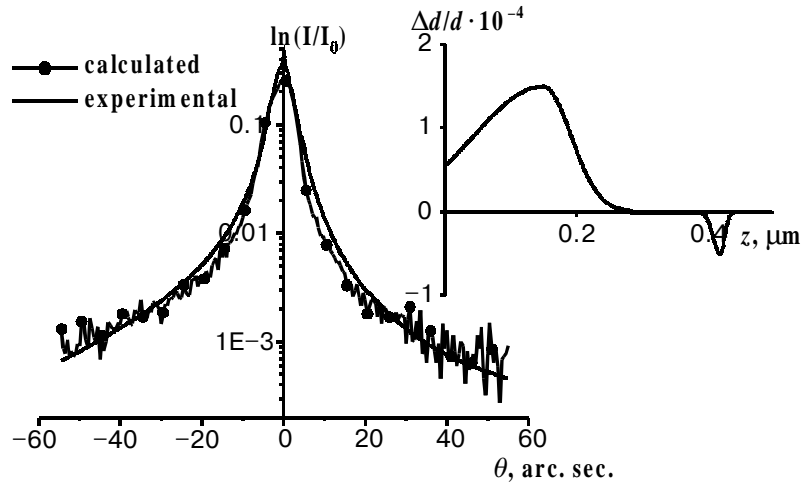

Fig. 2. Experimental and calculated rocking curves for the part №2. (333) reflection of $\mathrm{CuK}_{\alpha}$-radiation. The profile of strains shown on the right

in comparison with the initial part of the sample. The halfwidth of X-ray rocking curves and maximum values of intensity slightly increase. The general view of rocking curves indicates that there are near-surface layers with various levels of distortions, extensions and strain distributions in the treated parts of sample. These parts are characterized by expanded alternating-sign strains $(\varepsilon)$ in the direction perpendicular to the surface. The parameters of strains in different parts are:

№2: depth extension of strains is $L \sim 0.44 \mu \mathrm{m}$, the greatest positive strain is $\varepsilon_{\max }^{+} \sim 1.5 \cdot 10^{-4}$ at $z_{\max }^{+}=$ $=0.15 \mu \mathrm{m}$, the greatest negative strain is $\varepsilon_{\max }^{-} \sim$ $\sim-0.5 \cdot 10^{-4}$ at $z_{\max }^{-}=0.42 \mu \mathrm{m}$;

№3: $L \sim 0.6 \mu \mathrm{m}, \varepsilon_{\max }^{+} \sim 1 \cdot 10^{-4}$ at $z_{\max }^{+}=0.17 \mu \mathrm{m}, \varepsilon_{\max }^{-} \sim$ $\sim-0.2 \cdot 10^{-4}$ at $z_{\max }^{-}=0,4 \mu \mathrm{m}$.

№4: $L \sim 0.58 \mu \mathrm{m}, \varepsilon_{\max }^{-} \sim-0.2 \cdot 10^{-4}$ at $z_{\max }^{-}=0.12 \mu \mathrm{m}$ with the following strain decay according to the exponential law to a depth of $0,34 \mu \mathrm{m}$, and next $\varepsilon_{\max }^{-} \sim 0.2 \cdot 10^{-4}$ at $z_{\max }^{-}=0.43 \mu \mathrm{m}$.

It should be noted that another structure defects can influence on real values of strains, for example, tetrahedral packing defects of the vacancy type, dislocation loops of the interstitial type [14] as well as compression of a crystal lattice owing to formation of a various sort precipitations such as $\mathrm{SiC}$.

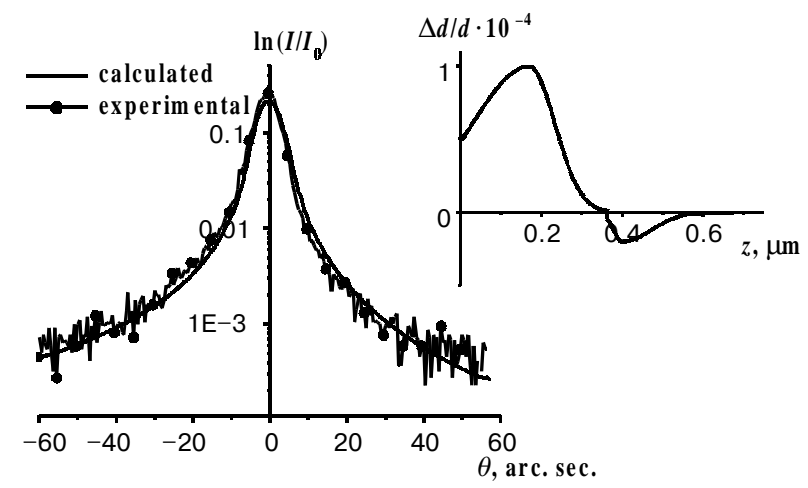

Fig. 3. Part №3. Notations are similar to those in Fig.2.

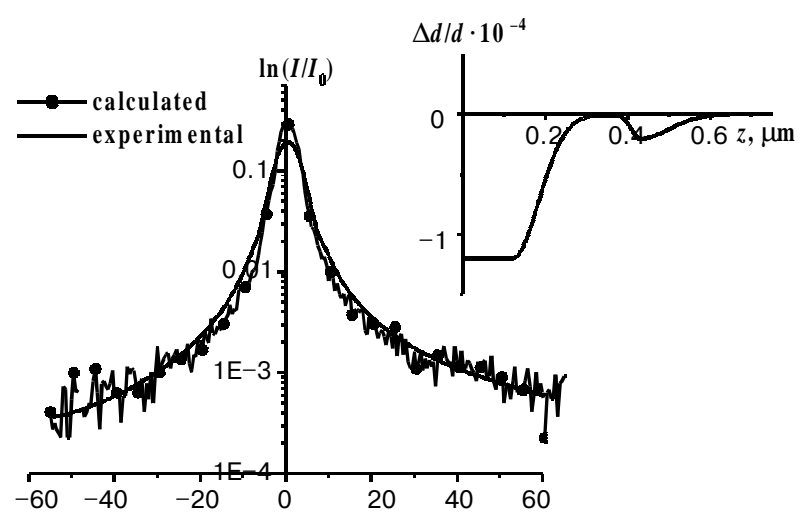

Fig. 4. Part №4. Notations are similar to those in Fig. 2.

According to data of atomic force microscopy (Fig. 1) the cross-sections of silicon filaments and isolated parts (clusters) after chemical etching (part №4) had minimal sizes of the order of several nanometres. Therefore, surface distortions are insignificant as judged from both changes of interplanar spacing and strain extension. It is confirmed by the behaviour of "tails" on the experimental rocking curves (Figs 3-4). The main contribution to the increase of the diffusion component of X-ray scattering on rocking curve "tails" is made, apparently, by the change of the surface layer density (degree of porosity is $\sim 40 \%$ ) and by the surface relief.

As would be expected, the greatest value of average deviation of heights from the mean plane of the surface relief $R_{a}=0.398 \mathrm{~nm}$ is inherent to the sample part №3. In the parts №2 and №4, values of $R_{a}$ are $0.288 \mathrm{~nm}$ and $0.303 \mathrm{~nm}$, respectively. The initial part of the crystal is characterized by the lowest value $R_{a}=0.17 \mathrm{~nm}$.

\subsection{Photoluminescence}

The analysis of photoluminescence spectra obtained over the interval of 24 months was carried out to amplify the investigations of structural features of all the parts of this porous silicon sample. Under action of light generated luminescence the spectral bands of luminescence shifted into the red area for sample parts №3 and №4 (Fig. 5). This shift occurred due to intensity redistribution between different bands. According to [7], two components in the spectrum correspond to two groups of silicon filaments, which differ one from another by the filament diameter. In other words, there is a dependence of the energy (wavelength) of recombination radiation on the structural features of the porous part. Taking into account the fact that longwave component of spectrum reduces more slowly for porous parts in comparison with the initial part the relative intensity of this component in the spectrum grows, as confirmed by experimental data.

As a result of ageing of porous silicon, the broadening (by $\sim 30 \%$ ) of the main band and insignificant in- 


\section{Z. Swiatek et al.: The influence of ion implantation by phosphorous on ...}
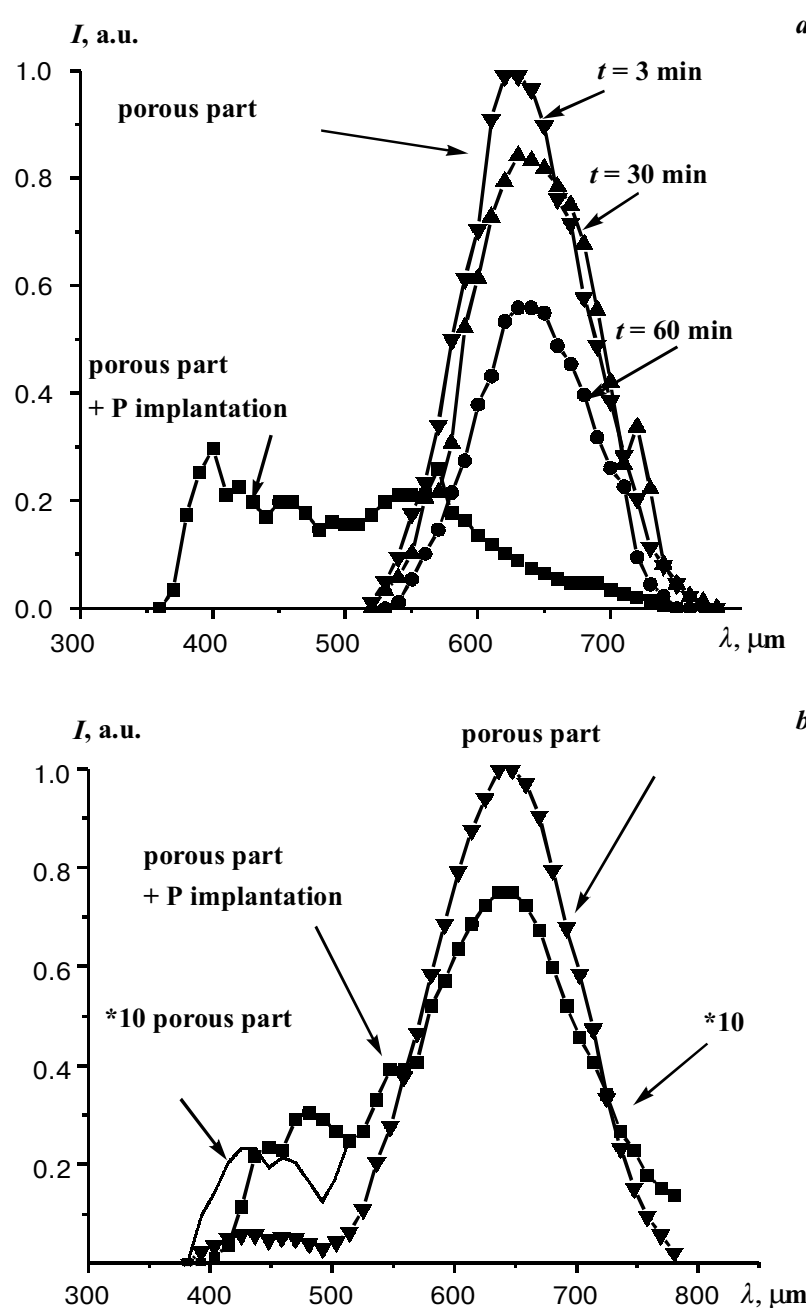

Fig. 5. Photoluminescence spectra from different parts of silicon sample: $a$ ) just treated sample, $b$ ) after 24 months.

crease (by $2-3 \%$ ) of intensity maximum were observed. The displacement of intensity to the longwave area and appearance of peak peculiar to porous silicon were revealed for the porous part implanted by phosphorus.

The effect of luminescence "fatigue" - autocratic reduction of the photoluminescence intensity at the same conditions of excitation - was observed in the obtained spectra (Fig. 5b). For 60 min, the intensity was reduced by half, and spectral band of luminescence was shifted by $25-35 \mu \mathrm{m}$. According to the majority of modern models, the spectral position of the visible luminescence band from porous silicon depends on the cross-section of silicon filaments with quantum sizes [1-7]. The reason of photoluminescence "fatigue" consists in the change of concentration of the centres of non-radiating recombination. The same concentrations of non-radiating centres cause a different probability of recombination for different band components. The time and spectral positions of band components are determined by structural features of porous layer, namely, by adsorbed molecules and various surface defects that cause randomized potential. The influence of this potential on the carriers drift depends on cross-sections of quantum silicon filaments.

\section{Conclusions}

1. The quantitative differences of thickness dependences of strains, values of the maximal strain, distortion level and extension of elastic strains were revealed for the parts of silicon sample subjected to implantation by phosphorus ions and chemical etching.

2 . The significant alternating-sign strains in direction perpendicular to the surface are peculiar to treated parts of silicon sample: $\varepsilon \sim 1.5 \cdot 10^{-4}$ for part №2, $\varepsilon \sim 10^{-4}$ for №3, $\varepsilon \sim-1.2 \cdot 10^{-4}$ for №4. The extension of strains in crystal depth is $300 \mathrm{~nm}$ and maximum of negative strains localised at the depth of $\sim 400 \mathrm{~nm}$.

The average heights of roughnesses $\left(R_{a}\right)$ of characteristic surface for the parts №1-4 are $0.17 \mathrm{~nm}, 0.288 \mathrm{~nm}$, $0.398 \mathrm{~nm}$, and $0.303 \mathrm{~nm}$, respectively.

3. The porous layer obtained on silicon surface and modified with previous implantation by phosphorus ions is characterized by an essentially different photoluminescence spectrum in comparison with the spectrum from the usual porous layer. The changes of the spectrum intensity as well as the displacement of the spectral band to longwave area are observed for porous parts of silicon sample under conditions of natural ageing for 24 months.

\section{References}

1. D. Buttard, G. Dolino, D. Bellet // Solid State Communications, №109. pp. 1-5 (1999).

2. E.V. Astrova, V.V. Ratnikov, R.F. Vitman // FTP, 31(5) pp. 1261-1268 (1997).

3. D.N. Gorechev, L.V. Belekov, O.M. Sreseli // FTP, 34(6). pp. 1130-1134 (2000).

4. E.V. Astrova, T.N. Vasyunkina // FTP, 36(5). pp. 593-596 (2002).

5. S.P. Zimin // FTP, 34(3). pp. 359-363 (2000).

6. M.E. Kompan, V.E. Hartsiev, I.Yu. Shabanov // FTT, 39(12), pp. 2137-2140 (1997).

7. U. Rissel, X. Ruge, Ionnaya implantatsiya. M.: Science, 1983. $234 \mathrm{p}$.

8. Z.T. Kuznitskiy // Neorganicheskiye materialy. 33(2). pp. 142146 (1997).

9. A.G. Kazanskiy, S.M. Petrushko, N.V. Rigov // FTP, 33(3) pp. 332-335 (1999).

10. V. Chamard, C. Pichat, G. Dolino // Solid State Communications, 118, pp. 135-139 (2001).

11. M. Servidori, F. Gembali // J. Appl. Cryst., 21, pp.176-181 (1988).

12. M. Nemiroff, V.S. Speriosu // J. Appl. Phys., 58(10) (1985).

13. A.V. Goncharskiy, A.V. Kolpakov, A.A. Stepanov // Metrologiya, 11, pp. 19-23 (1986).

14. S.A. Stepanov, E.A. Kondrashkina, A.N. Chuzo // Poverhnost, 9. pp. 112-118 (1988).

15. R. Fabbri, G. Lulli, R. Nipoti, M. Servidori // Nuclear Instruments and Methods in Physics Research B80/81, pp. 624-627 (1993).

16. I.M. Fodchuk, O.S. Kshevetskiy // Metallofizika, 14(5), pp. 57-62 (1992).

17. S.I. Geludeva, B.G. Zaharov, M.V. Kovalchuk, V.G. Kon, E.A. Sozontov, A.I. Sosfenov // Crystallographiya, 33(6). pp. 1352-1356 (1988). 